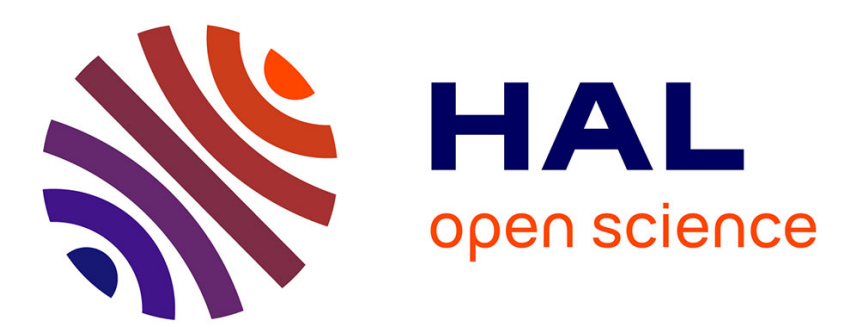

\title{
Non network-former cations in oxide glasses spotted by Raman scattering
}

\author{
Bernard Hehlen, Daniel R. Neuville
}

\section{To cite this version:}

Bernard Hehlen, Daniel R. Neuville. Non network-former cations in oxide glasses spotted by Raman scattering. Physical Chemistry Chemical Physics, 2020, 22 (22), pp.12724-12731. 10.1039/d0cp00630k . hal-02989584

\section{HAL Id: hal-02989584 https://hal.science/hal-02989584}

Submitted on 12 Nov 2020

HAL is a multi-disciplinary open access archive for the deposit and dissemination of scientific research documents, whether they are published or not. The documents may come from teaching and research institutions in France or abroad, or from public or private research centers.
L'archive ouverte pluridisciplinaire HAL, est destinée au dépôt et à la diffusion de documents scientifiques de niveau recherche, publiés ou non, émanant des établissements d'enseignement et de recherche français ou étrangers, des laboratoires publics ou privés. 


\title{
Non network-former cations in oxide glasses spotted by Raman scattering
}

\author{
Bernard Hehlen, ${ }^{* a}$ and Daniel R. Neuville, ${ }^{b}$ \\ The depolarized Raman spectra of about 30 binary and ternary aluminosilicate glasses have been \\ collected. Two spectral responses involving the cations are observed below $400 \mathrm{~cm}^{-1}$. One of the \\ two bands arises solely from cations close to non-bridging oxygens. This observation holds for all \\ the glasses investigated providing thereby a simple test for qualifying the polymerization state of \\ oxide glasses. The second feature involves all cations whatever their role in the glass and is found \\ to be twofold: one contribution arises form cations charge compensating $\mathrm{AlO}_{4}^{-}$tetrahedra and the \\ other one by network modifier cations. These results confirm the net vibrational contrast of cations \\ depending on their structural surrounding. Finally, while alkali atoms vibrate independently from \\ the rest of the network, alkali-earth cations close to non-bridging oxygens drag the latter in their \\ motion.
}

\section{I. Introduction}

Raman scattering is very sensitive to structural modifications in materials and provides in principle a powerful tool for probing the atomic structures. In disordered solids however the vibrational responses are usually broad, dissymmetric, and sometimes overlapping. In addition, the structural disorder often prevents for a selection rule analysis similar to that developed for crystals and molecules. Glasses suffer from these spectroscopic limitations which considerably limit the spectral analyses. Despite, there exists spectral indicators in Raman which provide valuable information on local and medium range structure, some being hardly accessible otherwise. In simple oxide glasses, these are for example the number of threefold and fourfold Si-O-Si rings $s^{1-4}$, the distribution of $\mathrm{SiO}_{4}^{(4-n)-}$ tetrahedra (so-called $\mathrm{Q}^{n}$ species) ${ }^{5}+7$, the Si-O-Si angle distribution in silicates $\frac{1448}{14}$, the amount of $\mathrm{BO}_{3}$ triangles and $\mathrm{BO}_{4}$ tetrahedra in botates 9 , among others. Very often though, relating the Raman data to structural numbers deserves calibration with a third party experiment such as NMR, X-Ray diffraction, or numerical simulations.

Vibrations involving the cations are located at low frequency, below $400 \mathrm{~cm}^{-1}$ typically and by now have been studied mostly

\footnotetext{
a Address, Laboratoire Charles Coulomb (L2C), UMR 5221 CNRS-Université Montepllier, Montpellier, France; Tel: $+334467 \quad 14 \quad 34$ 64; email : bernard.hehlen@umontpellier.fr

${ }^{b}$ Address, Géomatériaux, IPGP-CNRS, Université de Paris, 1 rue Jussieu, 75005 Paris, France.
}

by infrared spetroscopies ${ }^{10}-14$. Interestingly, there exists very few reports on Raman scattering, althought cations play a crucial role in the glass, for example when considering the melting temperature, the diffusion processes, the mechanical properties... From a structural point of vu, the cations (M) depolymerize the Si-O-Si connected network by creating ionic bonds with alkali and alkaline-earth units, in which the oxygen ions are called non-bridging oxygens (NBO). In aluminosilicates, when $\mathrm{Al}$ is in fourfold coordination it acts as a network former by forming $\mathrm{AlO}_{4}^{-}$tetrahedra covalently connected to the silica network. The cations can therefore be either network modifiers as in the previous case, or charge compensators when they compensate the negative charge carried by the $\mathrm{AlO}_{4}^{-}$tetrahedra. The balance between these two distinct functions varies as a function of the $\mathrm{M} / \mathrm{Al}$ ratio and controls many of the physical properties of the glass, such as the viscosity.

In a previous report we show that the depolarized Raman spectra of alumino-silicate glasses exhibit two cation bands: a low frequency one around $150 \mathrm{~cm}^{-1}$ hereafter called $\omega_{1}$ and a high frequency one around $330 \mathrm{~cm}^{-1}$ hereafter referred to as $\omega_{2} \cdot \frac{15}{15}$ In the two glass families investigated, $\omega_{2}$ involved only cations at modifier place, while all cations where supposed to contribute to $\omega_{1}$. The atomic displacements underlying these modes are not yet identified. However, a visual inspection of the vibrations calculated using first principle molecular dynamic simulations suggests that the latter corresponds to a dangling of the $\mathrm{Na}$ atom around the oxygen atom to which it is attached 16 . 
The aim of the present work is to investigate a large set of alkali and alkali earth silicate glasses in order to get more insights into the vibrational motions underlying the cations bands, enabeling conclusions which apply over the whole aluminosilicate glass family. To reach this objective, we investigated more than 30 glass compositions, the most prominant series being sets of alcalineearth aluminosillicates with constant silica content, $50 \mathrm{SiO}_{2}-z \mathrm{MO}-$ $y \mathrm{Al}_{2} \mathrm{O}_{3}$ and $\mathrm{M}=\mathrm{Mg}, \mathrm{Ca}, \mathrm{Sr}, \mathrm{Ba}$, with $y$ varying between 0 to 40 in mole \%. The ratio $R=\mathrm{MO} / \mathrm{Al}_{2} \mathrm{O}_{3}$ ranges from 0.25 to infinity and therefore crosses the tectosilicate join at $R=1$ (Table 1). The glasses contain both modifier and charge compensator cations for compositions in the paralcaline domain $(R>1)$, and only charge compensator cations for compositions in the peraluminate region $(R<1)$, in a simple model assuming all aluminum atoms fourfold coordinated. Sodo-silicates and sodo-aluminosilicates have also been studied to complement these series with alkali-based glasses. The compositions are summarized in Table 1.

Spectroscopic details and sample preparation are shortly described in section II. The spectroscopy in the binary glasses is presented in section III while ternary systems are discussed in detail in section IV. A special attention has been given to the low frequency cation mode which turned to be twofold in the aluminosilates glasses.

\section{II. Experimental conditions and sample preparation}

The Raman spectra have been recorded using a single pass HR630 Jobin-Yvon diffractometer. The incident light was delivered by a solid state laser working at $532 \mathrm{~nm}$ with a linearly polarized beam focused into the sample with a $\times 100$ microscope objective. The polarization of the light scattered in the backscattering geometry was analysed by a half plate wave followed by a broad band Glan-Thomson polarizer while the strong elastic line was rejected using two Bragg filters 17 . To ensure a good reproducibility of the scattered intensities, the irradiated surface of the samples were all flats and polished to optical quality. Finally all spectra were recorded after each other keeping the same experimental conditions.

The samples were obtained by melting mixtures of $\mathrm{Na}_{2} \mathrm{CO}_{3}$, $\mathrm{CaCO}_{3}, \mathrm{SrCO}_{3}, \mathrm{BaCO}_{3}, \mathrm{MgO}, \mathrm{Al}_{2} \mathrm{O}_{3}$ and $\mathrm{SiO}_{2}$ (Rectapur from Merck). The mixing was ground for 1 hour under alcohol in an agate mortar, heated slowly to decompose the carbonates, and then heated above the melting point following 18 . The melts were maintained for a few hours at high temperature (1900K for aluminosilicate melt and $1400 \mathrm{~K}$ for silicate melt) in air. The sample was quenched in a few seconds from high temperature by dipping the bottom of the platinum crucible into pure water. Peraluminous glasses were obtained by melting a ceramic firstly made at high temperature following previous paper 21 respectively for $\mathrm{Ca}-, \mathrm{Mg}$-, and Sr-aluminosilicate glasses). The heating procedure was repeated until no crystallization could be detected by optical microscope and X-ray diffractometry. All materials were found to be chemically homogeneous glassy phases before and after viscosity measurements. Glass sample were analyzed using an electron microprobe analyses, Cameca SX100.

\section{III. Binary glasses}

\subsection{Sodo-silicates: fitting strategy}

For sake of data homogeneity, the Raman spectra of the sodosilicates investigated in ${ }^{15}$ were recorded again using the same experimental conditions as for all the other glasses studied in this work. The depolarized Raman spectra shown in Figure 1 provides

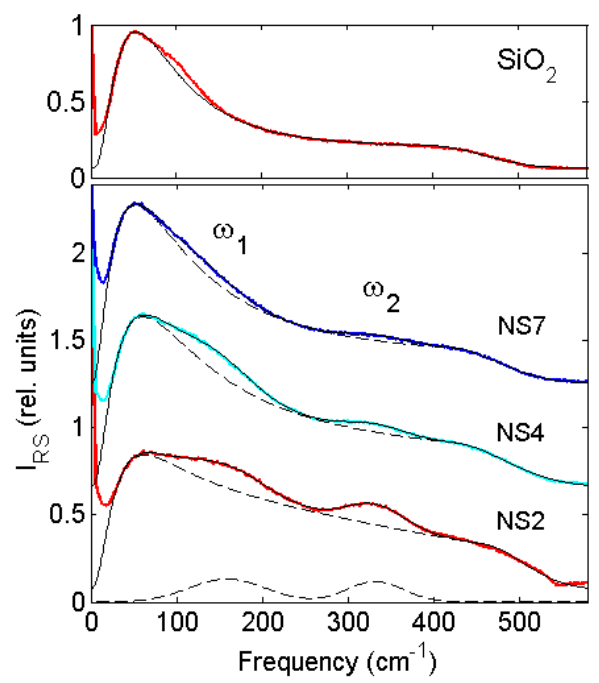

Fig. 1 Depolarized Raman spectra $\left(l_{V H}\right)$ of selected sodosilicate glasses and pure silica in the region of the cations modes $\omega_{1}$ and $\omega_{2}$. The solid lines result from a fit using a modified lognormal function for the boson peak (see text) and gaussians for the cation modes $\omega_{1}$ and $\omega_{2}$ (dashed lines).

examples for describing the fitting procedure. Extracting information on the cation modes deserves fitting simultaneously the boson peak (BP) and its decay at high frequency. The steep increase up to its maximum arises from quasi-local optic vibrations competing with a continuum of acoustic-like excitations. Above the maximum, incoherent scattering from optic modes possibly comes into play giving rise to a sample dependent response.22/23 Whatsoever the origin, there exists no analytical way to describe this complex spectral shape and one therefore has to find acceptable compromises. In our former paper ${ }^{15}$ this signal was modeled by an exponential decay in the frequency region surrounding the high frequency cation mode $\omega_{2 m}$ around $330 \mathrm{~cm}^{-1}$. We now want to analyse $\omega_{1}$ at low frequency together with $\omega_{2}$. This requires fitting a larger spectral range and hence the boson peak as a whole. The model chosen for fitting the boson peak is composed by a lognormal function accounting for its low frequency part, an exponential decay taking over continuously at frequencies above 
Table 1 List of glass samples and location in the ternary phase diagram.

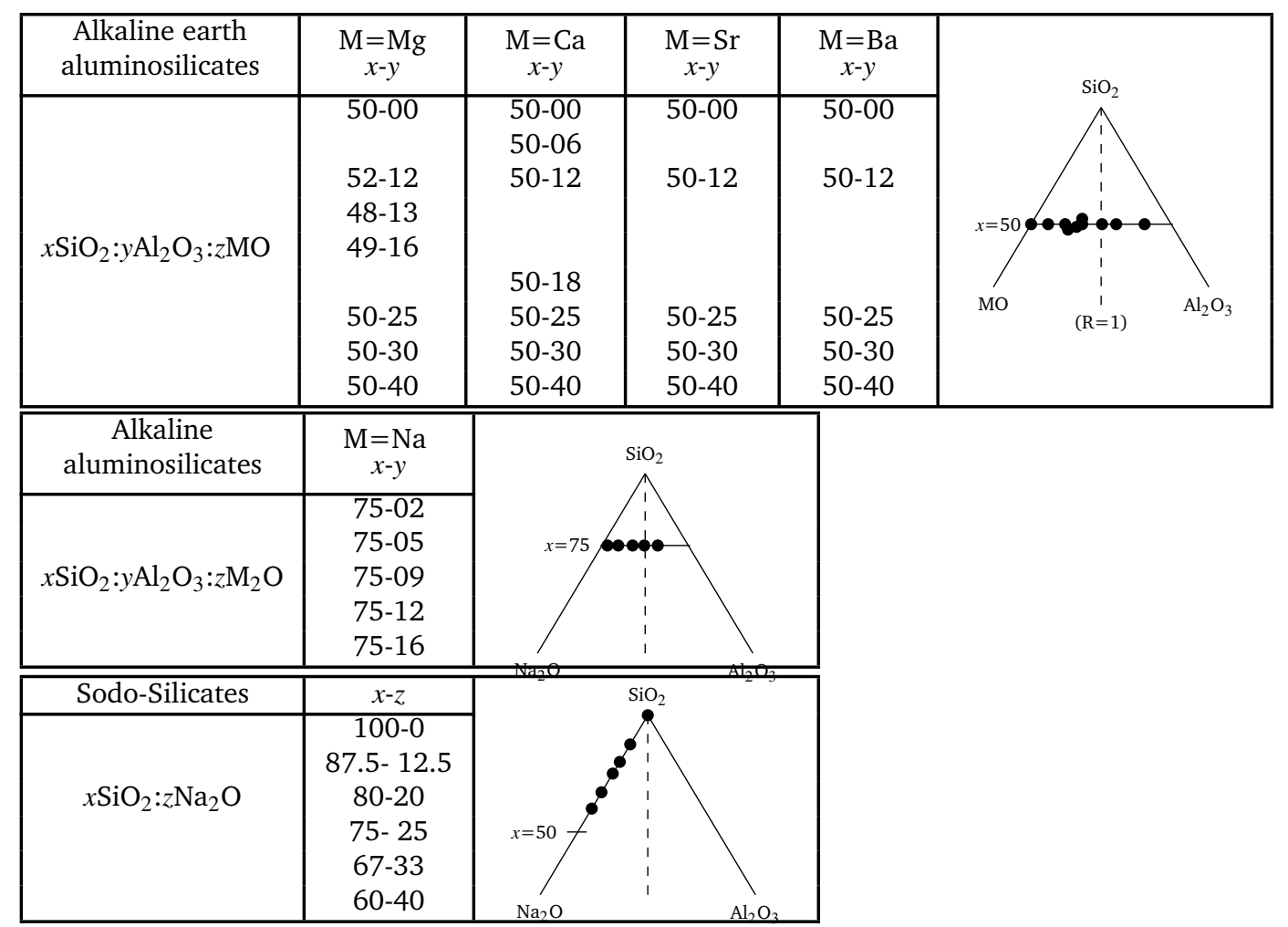

its maximum (above $\sim 150 \mathrm{~cm}^{-1}$ typically), and a cutoff with a gaussian decay shape around $450-500 \mathrm{~cm}^{-1}$. This "modified lognormal" function is clearly a rough approximation, but it has the merit of being relatively stable during the fitting procedure, with fitting parameters evolving smoothly and monotoneously from one spectrum to the other, reproducing thereby the evolution of the boson peak. The cation modes $\omega_{1}$ and $\omega_{2}$ on top of this signal have been modeled by gaussian functions characterized by their frequency, width, and area. The numerous fitting tests we performed reveal that the output parameters of $\omega_{2}$ are quite robusts. The reason is that it stands on a relatively flat background in all glasses. The steep and curved slope on which stands $\omega_{1}$ leads to larger uncertainties, sometimes limiting the spectral analysis.

The superposition of the model (solid line) with the experimental data in sodosilicates (Fig. 1) highlights the good quality of the fits, at least for that glass series. Despite, in pure silica the modified lognormal function is unable to reproduce the bump around $120 \mathrm{~cm}^{-124}$. For some glasses this peculiar shape of the BP is an additional drawback limiting the fitting quality. Therefore, we will only present below the trends of the fitting parameters which are not (or weakly) sensitive to the fitting quality of the boson peak "background" signal. The only exception is the area of $\omega_{1}$, subject to large uncertainty, but whose behavior turns out to be useful for the discussion. It is finally worth noticing here that the
IR response of $\omega_{1}$ is most likely bimodal $\frac{11 \mid 12}{12}$ but still, we fitted its Raman signature with a single gaussian according to the fact that the proximity of the strong boson peak prevents from a more detailed analysis.

\subsection{Alkali earth silicates}

$\omega_{1}$ and $\omega_{2}$ are also present in alkali-earth silicates as shown in the depolarized Raman spectra of the $50 \mathrm{SiO}_{2}: 50 \mathrm{MO}$ glasses with $\mathrm{M}=\mathrm{Mg}, \mathrm{Ca}, \mathrm{Sr}$, and $\mathrm{Ba}$ (Figure 2). The responses are intense and can also be observed in experiments performed without polarization analysis. In the latter situation however, the Si-O-Si bending motions (R-band) together with the signal arising from the boson peak drastically confuse the spectral analysis. The arrow in Figure $2 \mathrm{a}$ indicates the position of $\omega_{2}$ in $\mathrm{MgSiO}_{3}$ extrapolated from the three other glasses. It shows that $\omega_{2}$ is absent (or very weak) in that glass while very strong in the three other compounds. This observation provides an additional evidence that $\mathrm{Mg}$-based silicates behave in a peculiar way as compared to other silicates. For example, they exhibit a highly disordered local structure with a coordination number of $\sim 4$ to $4.5^{20|25| 26}$ significantly lower than for the other alkaline earth cations, between 6 and 8 . These effect correlate with the value of the field strength which is the highest for magnesium, bringing the authors to sometimes position this cation as intermediate between glass modifier and glass former. 


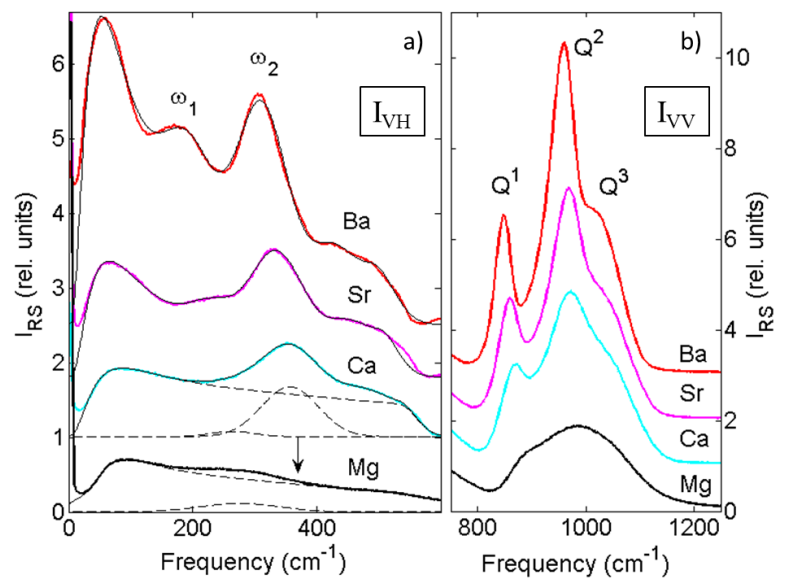

Fig. 2 a) Depolarized Raman spectra $\left(\mathrm{I}_{V H}\right)$ of $\mathrm{MSiO}_{3}$ glasses (50MO:50SiO ${ }_{2}$ ) with $\mathrm{M}=\mathrm{Mg}, \mathrm{Ca}, \mathrm{Sr}, \mathrm{Ba}$, and their fits. The arrow indicates the position of $\omega_{2}$ in $\mathrm{MgSiO}_{3}$ extrapolated from the three other glasses. b) Polarized Raman spectra $\left(I_{V V}\right)$ : zoom on the $\mathrm{Q}^{n}$ bands. For sake of visibility, the spectra have been translated vertically.

These structural specificities are also captured in the Raman response of the $\mathrm{Q}^{n}$ species shown in Figure 2 . The $\mathrm{Q}^{2}$ and $\mathrm{Q}^{1}$ bands are rather well defined except in the $\mathrm{Mg}$-based glass where the $\mathrm{Q}^{n}$ feature transforms into a single and very broad structure. This behavior likely results from a pronounced structural disorder at the Si-NBO sites giving rise to ill-defined Si-NBO stretching motions. Finally, the strong disorder close to the $\mathrm{Mg}$ cation also explains the very broad response of $\omega_{1}$ in $\mathrm{MgSiO}_{3}$ (Fig.21) as compared to other $\mathrm{MSiO}_{3}$ glasses.

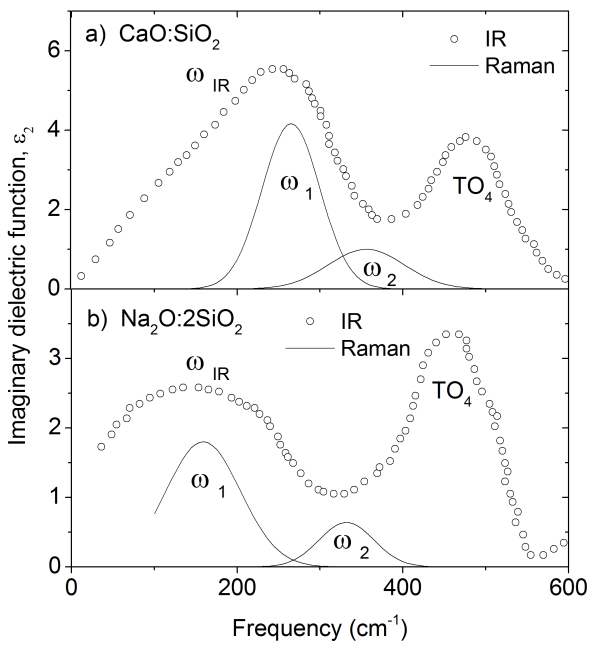

Fig. 3 Comparison between the dielectric function $\varepsilon_{2}$ measured in IR (circles) and the response of the two cations bands $\omega_{1}$ and $\omega_{2}$ extracted from the Raman spectra (lines, see text for details) in a) $\mathrm{CaO}_{\mathrm{SiO}_{2}}{ }^{13}$ and b) $\mathrm{Na}_{2} \mathrm{O}: 2 \mathrm{SiO}_{2} 10$.

Figure 3 compares the Raman signature of the cation modes $\omega_{1}$ and $\omega_{2}$ with the IR spectra for the binary compounds $\mathrm{CaO}: \mathrm{SiO}_{2} \underline{13}$ $\left(\mathrm{CaSiO}_{3}\right)$ and $\mathrm{Na}_{2} \mathrm{O}: 2 \mathrm{SiO}_{2}{ }^{10}\left(\mathrm{Na}_{2} \mathrm{Si}_{2} \mathrm{O}_{3}\right) . \omega_{2}$ falls in the deep between the broad feature $\omega_{I R}$ assigned to cation motions ${ }^{12}$ and the transverse optic mode TO4 around $480 \mathrm{~cm}^{-1}$ corresponding to the rocking of the $\mathrm{Si}-\mathrm{O}-\mathrm{Si}$ bonds ${ }^{27}$. $\omega_{1}$ has a frequency similar to that of $\omega_{I R}$ suggesting that both responses correspond to the same type of vibrations. For the reasons mentioned in the preceding section (lack of accuracy owing to the strong boson peak nearby), we prefered to fit $\omega_{I R}$ with one single gaussian although the IR data clearly show a structured spectral shape du to different cation environments motivating fitting procedures using two gaussians 12 .

For a harmonic oscillator involving $n$ atoms, like e.g. a molecule, the eigenfrequencies are given by $\omega_{0}=\sqrt{k_{c} / \mu}$ where $k_{c}$ is the caracteristic spring constant and $\mu$ the reduce mass of the eigenmode under consideration. The frequency of the two IR components building the infrared response $\omega_{I R}$ is reproduced in Figure 4. It exhibits a linear behavior when plotted as a function of $\sqrt{1 / m_{\mathrm{M}}}$, where $m_{\mathrm{M}}$ is the mass of the cation 12 . Since $\mu=m_{\mathrm{M}}$, this lead the authors to conclude that the modes building $\omega_{I R}$ involve solely the cations, i.e. in a motion almost independent from their anionic surrounding.

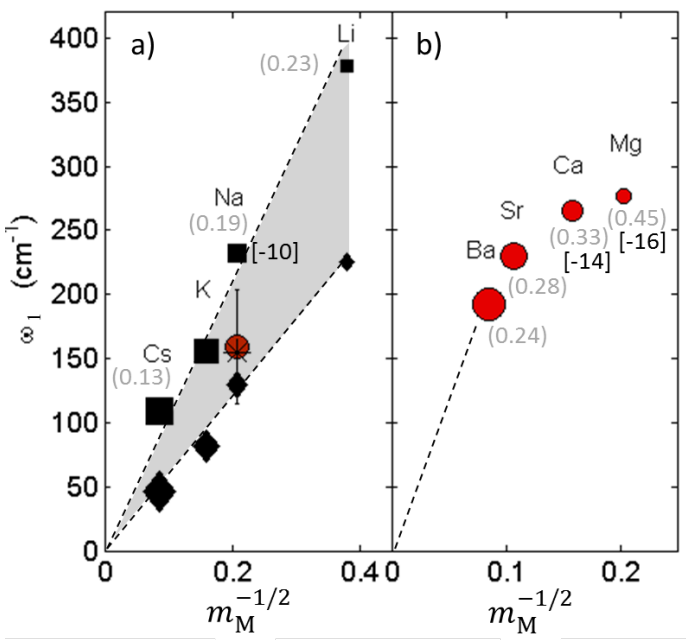

Fig. 4 Frequency of $\omega_{1}$ in Raman and $\omega_{I R}$ in Infrared plotted as function of $\sqrt{1 / m_{\mathrm{M}}}$. The size of the symbols refers to the atomic radii of the cations a) Alkaline-silicates: The circle is our work, the filled squares and filled losanges correspond to the two-component description of the IR response $\omega_{I R}\left[12\right.$, and the star is reproduced from ${ }^{10}$. b) Alkaline-earth silicates (our work). The numbers in parentheses are the field strengths 28 and those in braquets are $\log _{10}(D)$ where $D$ are the diffusion coefficients compiled from? .

Lets now consider the case of alkaline-earth silicates (Figure $4 \mathrm{p}$ ). The frequency $\omega_{1}$ now deviates from the linear behavior and the effect becomes more pronounced for lighter cations. This different behavior between alkali and alkali earth ions goes along with the increase of the atomic field strengths which are 

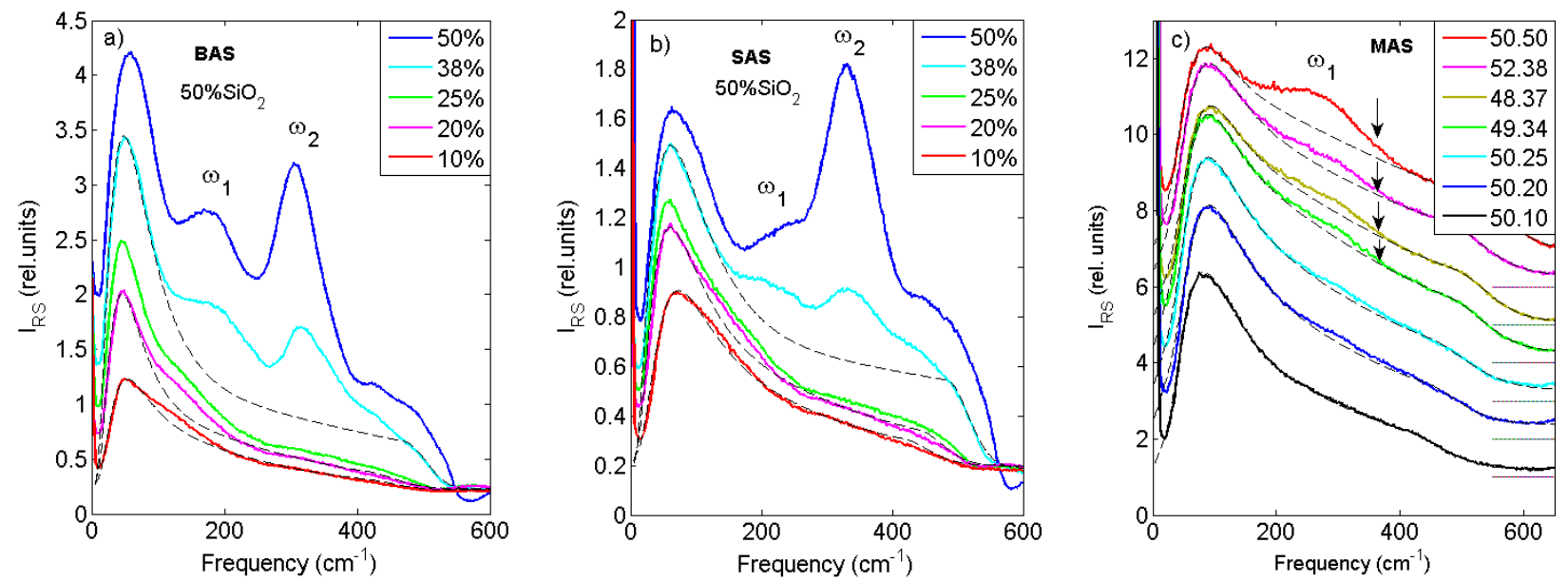

Fig. 5 Depolarized Raman spectra $\left(\mathrm{I}_{V H}\right)$ of aluminosilictes, $x \mathrm{SiO}_{2}: y \mathrm{Al}_{2} \mathrm{O}_{3}: z \mathrm{MO}$. a) $\mathrm{M}=\mathrm{Ba}$, with $x=50 \%$ and $z$ in the legend, b) $\mathrm{M}=\mathrm{Sr}$, with $x=50 \%$ and $z$ in the legend, c) $\mathrm{M}=\mathrm{Mg}$, with the legend in the format x.z. Examples of fitted boson peaks are shown to give an idea of the spectral shapes underneath $\omega_{1}$ and $\omega_{2}$ (dashed lines). The spectra in Mg-based glasses have been translated-up for sake of clarity. The baselines are given by the horizontal lines on the right. The arrows indicate the expected positon of $\omega_{2}$ in MAS.

higher for the former. Indeed, when the electrostatic interaction increases, the cation become more and more bonded to the oxygens nearby and drag them along in its motion. The mass of the vibrating structure increases and hence $m_{\mathrm{M}}$ should be replaced by $\mu$.

When considering $m_{\mathrm{M}}$ instead, the curve $\omega=f\left(\sqrt{1 / m_{\mathrm{M}}}\right)$ artificially bends down, exactly as $\omega_{1}$ does in Figure $4 \mathrm{p}$. The increasing network connectivity for light atoms goes along with a decrease of the cation diffusivity $D$, revealing in addition the close link between mode frequency, field strength, and diffusivity. This analysis captures the overall motions of the cations : two main vibrations in their cage, $\omega_{1}$ and $\omega_{2}$, and the relaxations at high temperature, i.e diffusion processes characterized by the diffusion coefficient $D$.

\section{IV. Aluminosilicates}

The depolarized Raman spectra of the ternary aluminosilicates series $x \mathrm{SiO}_{2}: y \mathrm{Al}_{2} \mathrm{O}_{3}: z \mathrm{MO}$ with $\mathrm{M}=\mathrm{Ba}, \mathrm{Sr}, \mathrm{Mg}$, are shown in Figure 5 . The spectra of calcium glasses are displayed in Figure $4 \mathrm{~b}$ of Ref.15. Surprisingly, the appearance of an additional structural surrounding for the cations in the ternary systems does not translate into additional Raman bands. However, a direct inspection shows that $\omega_{1}$ is present down to the lowest cation concentration suggesting that the band now gather the motions of both network modifier and charge compensator cations. One also observes that $\omega_{2}$ vanishes around $z=25$, that is at the joint $R=1$ ( $\mathrm{Ba}$ and $\mathrm{Sr}$ glasses) confirming previous anticipations that this band arises solely from cations close to non-bridging oxygens. Similarly to the binary $\mathrm{MgO}: \mathrm{SiO}_{2}$ system, $\omega_{2}$ is still absent (or very weak) in all of the $\mathrm{MgO}$-aluminosilicates investigated. For this set of glasses the silica content is close but not always equal to $50 \%$ and the spectra are displayed by decreasing concentration of $\mathrm{MgO}$ to highlight the concomitant intensity decrease of $\omega_{1}$.

\subsection{Low-frequency cation mode $\omega_{1}$}

Figure 6 gather the fitted integrated intensities of $\omega_{1}$ in the aluminosilicates and sodosilicates series. In calcium glasses $\omega_{1}$ is embedded under the strong $\omega_{2}$ band and the fitting parameters could not been exploited (see Fig. 23). For the other glasses, the relatively large dispersion of the points arises from the fitting limitations mentioned in section III. Despite, when scaling all the intensities on the same value at $z=25(R=1)$ one clearly observes a change in the slope between peraluminate and paralcaline domains. The effect is more pronounced for heavy cations and likely accounts for a change in the Raman efficiency when network modifiers $(R>1)$ or charge compensators $(R<1)$ are involved.

This and the step increase of the frequency of $\omega_{1}$ around $R=1$ shown in Figure 6p suggests a doublet structure of the Raman response : when all cations are charge compensators $([z]<25$, neglecting $\mathrm{Al}^{V}$ or $\mathrm{Al}^{V I}$ structures) the frequency $\omega_{1}\left(\equiv \omega_{1 c}\right)$ is fairly constant and has a different value than $\omega_{1}\left(\equiv \omega_{1 m}\right)$ for the binary glasses for which all cations are network modifiers ([z] $=50$ ). In between, the glasses have both network modifier and charge compensator cations, and the Raman response likely becomes twofold, i.e. $\omega_{1}=\omega_{1 c}+\omega_{1 m}$. Unfortunately, the spectroscopy is unable to separate the two contributions, leading to an apparent up-shift (or down-shift in Mg-based glasses) of the frequency when cation oxide is replaced by aluminum oxide. This 

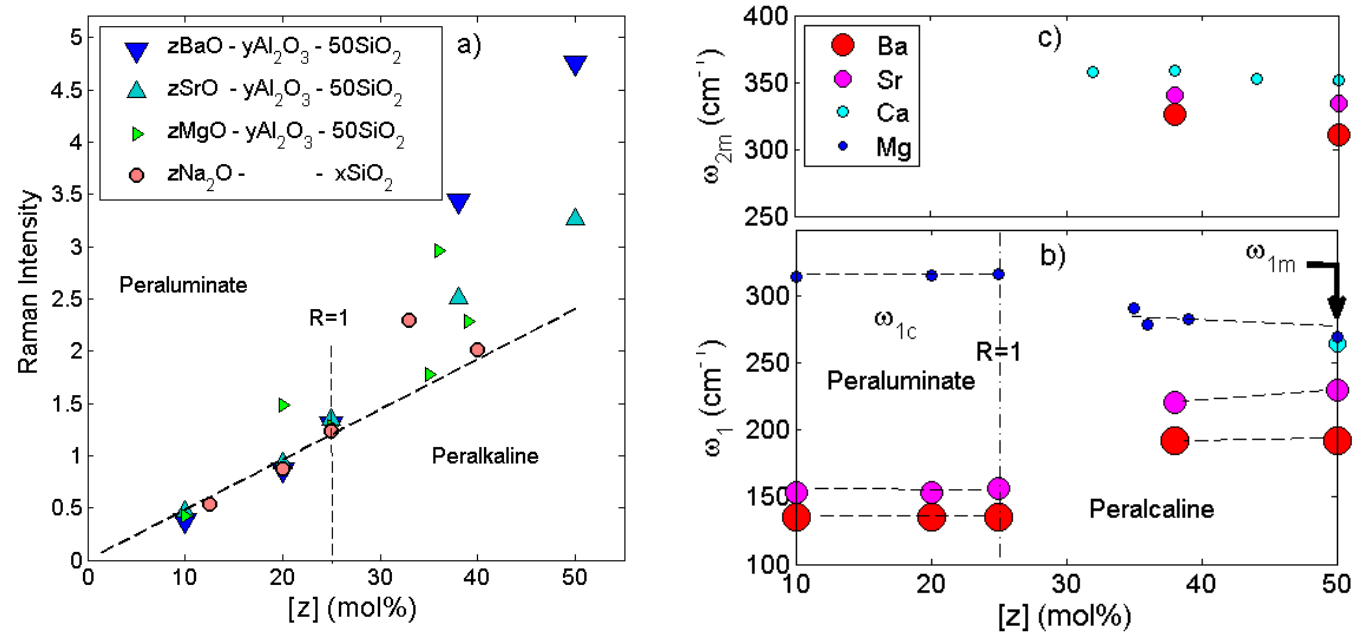

Fig. 6 a) Integrated intensity of $\omega_{1}$ as a function of the cation content [z]. The data have been scaled to coincide at concentration $z=25(R=1)$. b) and c) Frequency of the cations modes $\omega_{1}$ and $\omega_{2}$ as a function of $[z]$, respectively.

is however well correlated with the ${ }^{23} \mathrm{Na}$ NMR observed between sodium silicate and aluminosilicate glasses ${ }^{30}$ where a NMR chemical shift is observed between charge compensator and network modifier cations, and also with XANES results at the Ca K-edge of $\mathrm{CaO}-\mathrm{SiO}_{2}$ and $25 \mathrm{CaO}-25 \mathrm{Al}_{2} \mathrm{O}_{3}-50 \mathrm{SiO}_{2}$ glasses, where a shift in the XANES pre-edge is observed as a function of the cation site ${ }^{31}$.

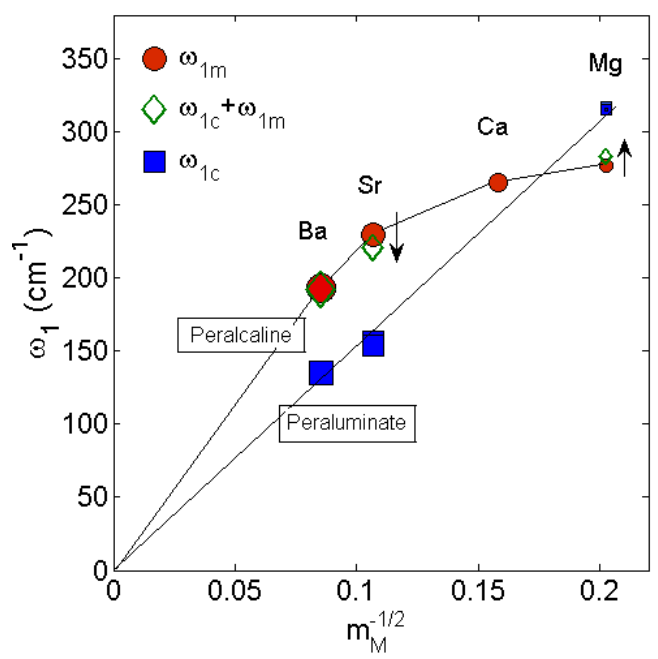

Fig. 7 Frequency of $\omega_{1}$ as a function of $\sqrt{1 / m_{\mathrm{M}}}$ in the aluminosilicate glasses. Red filled circles: binary glasses $\mathrm{MOSiO}_{3}([z]=0)$; green diamonds: paralkaline glasses $([z]=12 \%)$; blue squares: peraluminate glasses, $([z]=25 \%, 20 \%$ and $10 \%$, from dark blue to light blue). In the latter the frequency is similar for the three $[z]$ values and the points superpose. Lines are guide to the eye.

In an attempt to confirm the above assumption we gather in
Figure 7 the curves $\omega_{1}(\mathrm{M})$ as a function of $\sqrt{1 / m_{\mathrm{M}}}$ for cations concentrations $[z]=10,20,25,38$, and $50 \mathrm{~mol} \%$. The filled circles correspond to the binary silicates, $[z]=50 \mathrm{~mol} \%$, the empty losanges correspond to aluminosilicates with $[z]=38 \mathrm{~mol} \%$ (peralkaline domain), and the squares to aluminosilicates with $[z]=$ 25, 20 and $10 \mathrm{~mol} \%$ (peraluminate domain). One observes two distinct behaviors. For a predominance of network modifier cations in the glass (peralkaline domain), cation motion couples with the oxygen atoms nearby leading to the observed bending of the frequency dependence of $\omega_{1}$, as explained in the preceding section. The situation is very different for glasses in the peraluminate domain. In that case, there is only one possible site for the cations, i.e. charge compensators, and the three data sets merge in a single straight line meaning that the motion of those cations is now decoupled from the rest of the network. Indeed, the negative charge carried out by the $\mathrm{AlO}_{4}^{-}$to which the cation is attached is delocalized leading to a loose directional bonding. Keeping in mind that $\omega_{1}$ likely results from a dangling motion of the cations, this and the large mass difference between cations and tetrahedra can produce the observed "vibrational decoupling effect". However, although directionally loose, the bonding of the cation to its $\mathrm{AlO}_{4}^{-}$tetrahedra should remain strong along the radial axis, as indicated by the weaker value of the diffusion coefficient of charge-compensator cations as compared to network modifiers ones. This result is in line with the IR data of alkalineearth borate glasses ${ }^{32}$ where the authors also found a linear behavior in a plot of $\omega_{I R}$ versus $\sqrt{1 / m_{\mathrm{M}}}$. For the studied concentration (0.45MO:0.55 $\mathrm{B}_{2} \mathrm{O}_{3}$ ) most of the cations compensate $\mathrm{BO}_{4}^{-}$ tetrahedra ${ }^{33}$ which corresponds to a situation similar to the peraluminate domain in alumino silicates. Finally, Table 2 summarizes the values for these three sets of frequencies, i.e. $\omega_{1 c}, \omega_{1 m}$, and 
$\omega_{2}$

Table 2 Frequency of the two cation modes $\omega_{1}$ and $\omega_{2}$ in alkaline-earth aluminosilicates (in $\mathrm{cm}^{-1}$ ): contribution of charge compensator cations $\omega_{1 c}$ and network modifier cations $\omega_{1 m}$ to the low frequency response $\omega_{1}$, and high frequency response $\omega_{2}$ originating solely from modifier cations and taken at $[z]=50$.

\begin{tabular}{|c|c|c|c|}
\hline Cation (M) & \multicolumn{2}{|c|}{$\omega_{1}$} & $\omega_{2}$ \\
& $\omega_{1 c}$ & $\omega_{1 m}$ & \\
\hline $\mathrm{Mg}$ & 316 & 270 & - \\
$\mathrm{Ca}$ & - & 265 & 352 \\
$\mathrm{Sr}$ & 155 & 230 & 334 \\
$\mathrm{Ba}$ & 135 & 193 & 310 \\
\hline
\end{tabular}

\subsection{High frequency cation mode $\omega_{2}$}

Let's now consider the high frequency mode $\omega_{2}$. Its frequency and integrated intensity are presented as a function of the cation content in Fig. 6c and 8a, respectively. The latter compiles the data obtained in alkaline-earth aluminosilicates, sodo aluminosilicates, and sodo-silicates glasses. These families define three sets of data highlighted by blue (triangles), pink (filled circles), and yellow (empty circles) background colors. Within the alkalineearth group (triangles), the intensity of the three glass family has been assigned a multiplication factor and as a consequence, all curves superpose.

As expected, the intensities decrease to zero close to the joint $R=1$ in all of the ternary glasses. Considering only foufoldcoordinated $[\mathrm{Al}]^{I V}$ atoms in the glasses, this value is reached for $z=12.5$ in the sodium aluminosilicate, and $z=25$ in the alkali earth glasses (arrows in Fig. 8a). By multiplying all the sets by a constant accounting for the cation scattering efficiency, all the curves overlapp into a single one, at least when plotted as a function of $[\mathrm{z}]_{\text {mod }}$, the molar fraction of modifier cations (Fig. 8b). Within our structural approximation, each couple of $\mathrm{AlO}_{4}^{-}$ tetrahedra is charge compensated by one alkali earth atom, and the latter reads $[\mathrm{z}]_{\text {mod }}=(z-y) / \mathrm{atm}$, where atm is the number of atoms per mole. When $[\mathrm{z}]_{\bmod }$ becomes low, i.e. when the concentration of alumina increases up to the peraluminate region, the intensity of $\omega_{2}$ reaches zero before the joint $R=1\left([\mathrm{z}]_{\text {mod }}=0\right)$ in the alkaline-earth glasses, a behavior also visible in Figure 8a. This observation goes along with an increase of $[\mathrm{All}]^{V}$ and $[\mathrm{Al}]^{V I}$ environments. Compensating these structures requires additional cations which hence are lost as network modifiers, leading to a faster decrease of the intensity of the mode as compared to the linear regime extrapolated from high $[\mathrm{z}]_{\text {mod }}$ values (dashed line in Figure 8b). However, the effect is rather weak and the experimental errorbars prevent for a comparative analysis between $\mathrm{Ca}$, Sr, and Ba.

\section{Conclusion}

The Raman spectra of alkaline and alkaline-earth aluminosilicates exhibit two spectral responses involving cation motions. The first one at low frequency, typically around $150 \mathrm{~cm}^{-1}\left(\omega_{1}\right)$, is likely twofold though not resolved by our experiment. One component $\left(\omega_{1 c}\right)$ arises from cations compensating the negative charge carried by the $\mathrm{AlO}_{4}$ tetrahedra. Those cations vibrate independently to the heavy structure to whom they are attached. This decoupling is further amplified by the loose and delocalised electrostatic bonding. The second component $\left(\omega_{1 m}\right)$ involves cations at modifier's place, that is attached to non-bridging oxygens. In alkaline-earth alumino silicate glasses the cations drag the NBOs nearby in a coupled motion, a situation different than for alkali cations where the vibration is defined solely by the motion of the cation. A likely explanation is the difference of strength fields which are significantly higher for alkali earth cations. The second cation band appears around $330 \mathrm{~cm}^{-1}\left(\omega_{2}\right)$. In aluminosilicates the analysis shows that whatever the cation type, only those at modifier's place contribute to that vibrational response. Overall, these results point-out a clear vibrational contrast between the cations attached to NBOs and those close to $\mathrm{AlO}_{4}$ tetrahedra. The presence or not of $\omega_{2}$ in a Raman spectra also provides a simple test for qualifying the polymerization state of a glass. Since responses at similar frequencies appear in the Raman spectra of borate and borosilicate glasses these conclusions likely hold also in these systems. 

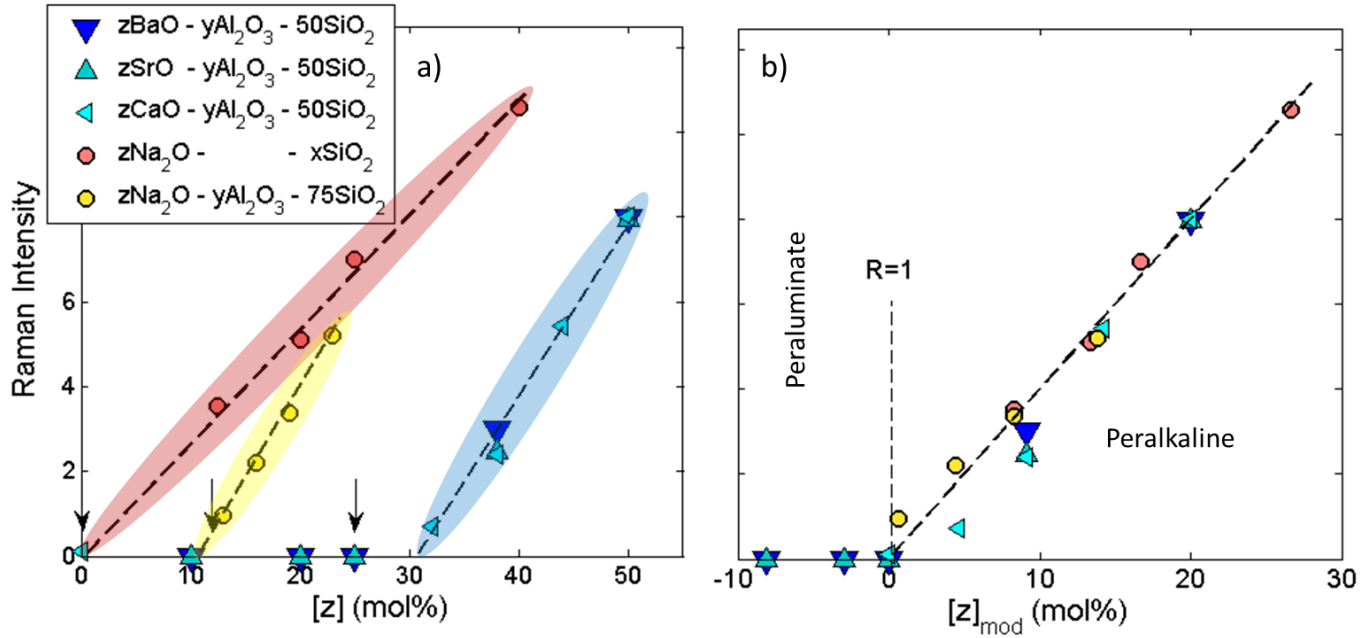

Fig. 8 Integrated intensity of $\omega_{2}$ a) as a function of the total cation content $[z]$ and $\mathbf{b}$ ) as a function of the modifier cation content $[z]$ mod . The arrows indicate the join $R=1$.

\section{Notes and references}

1 F.L. Galeener and J.C. Mikkelsen Jr, Vibrational dynamics in ${ }^{18} \mathrm{O}$-substituted vitreous $\mathrm{SiO}_{2}$. Phys. Rev. B, 1981, 23, 55275530 .

2 F.L. Galeener, Planar rings in glasses Solid State Commun., 1982, 44, 1037-1040.

3 A. Pasquarello and R. Car, Identification of Raman defect lines as signatures of ring structures in vitreous silica Phys. Rev. Lett., 1998, 80, 5145-5147.

4 B. Hehlen, Inter-tetrahedra bond angle of permanently densified silicas extracted from their Raman spectra. J. Phys.: Condens. Matter, 2010, 22, 025401.

5 P.F. McMillan, Structural studies of silicate glasses and meltsapplications and limitations of Raman spectroscopy. Am. Mineral., 1984, 69, 622-644.

6 B.O. Mysen and J.D. Frantz, Structure and properties of alkali silicate melts at magmatic temperatures. Eur. J. Mineral., 1993, 5, 393-407.

7 B.O. Mysen, Experimental, in situ, high-temperature studies of properties and structure of silicate melts relevant to magmatic processes. Eur. J. Mineral., 1995, 7, 745-766.

8 B. Hehlen, D.R. Neuville, D. Kilymis and S. Ispas, Bimodal distribution of $\mathrm{Si}-\mathrm{O}-\mathrm{Si}$ angles in sodo-silicate glasses $J$. NonCryst. Solids, 2017, 469, 39-44.

9 F. Angeli, O. Villain, S. Schuller, T. Charpentier, D. De Ligny, L. Bressel and L. Wondraczek, Effect of temperature and thermal history on borosilicate glass structure. Phys. Rev. B, 2012, 85, 054110

10 C.I. Merzbacher and W.B. White, Structure of $\mathrm{Na}$ in aluminosilicate glasses: A far-infrared reflectances spectroscopics study. Am. Mineral., 1988, 73, 1089.

11 E.I. Kamitsos, A.P. Patsis and G.D. Chryssikos, Infrared reflectance investigation of alkali diborate glasses. J. Non-Cryst. Solids, 1993, 152, 246.

12 E.I. Kamitsos and G.D. Chryssikos, Alkali sites in glass. Solid State Ionics, 1998, 105, 75-85.

13 D. De Sousa Meneses, M. Malki and P. Echegut, Optical and structural properties of calcium silicate glasses. J. Non-Cryst. Solids, 2006, 352, 5301.

14 D. De Sousa Meneses, M. Eckes, L. Del Campo, C.N. Santos, Y. Vaills and P. Echegut, Investigation of medium range order in silicate glasses by infrared spectroscopy. Vib. Spectrosc., 2013, 65,50 .

15 B. Hehlen and D.R. Neuville, Raman response of network modifier cations in alumino-silicate glasses. J. Phys. Chem. B, 2015, 119, 4093-4098.

16 D. Kilymis, S. Ispas, B. Hehlen, S. Peuget and J.-M. Delaye, Vibrational properties of sodosilicate glasses from first-principle calculations Phys. Rev. B, 2019, 99, 054209.

17 OptiGrate Corp.: http://www.optigrate.com.

18 D.R. Neuville, Viscosity, structure and mixing in (Ca, Na) silicate melts. Chem. Geol., 2006, 229, 28-42.

19 D.R. Neuville, L. Cormier and D. Massiot, Al speciation in calcium aluminosilicate glasses: A NMR and Raman spectrocospie. Chem Geol., 2006, 229, 173-185.

20 D.R. Neuville, L. Cormier, V. Montouillout, P. Florian, F. Millot, J.-C. Rifflet and D. Massiot, Structure of $\mathrm{Mg}$ and $\mathrm{Mg} / \mathrm{Ca}$ aluminosilicate glasses: ${ }^{27} \mathrm{Al} \mathrm{NMR}$ and Raman spectroscopy investigations. Am. Mineral., 2008, 93, 1721-1731.

21 A. Novikov, D.R. Neuville, L. Hennet, D. Thiaudière, Y. Gueguen and P. Florian, $\mathrm{Al}$ and $\mathrm{Sr}$ environment in tectosili- 
cate glasses and melts: viscosity, Raman and NMR investigation. Chemical Geology, 2017, 461, 115-127.

22 H. Mizuno, H. Shiba and A. Ikeda, Continuum limit of the vibrational properties of amorphous solids, Proc. Natl, Acad. Sci., 2017, 114, 055902.

23 Y.M. Beltukov, C. Fusco, D.A. Parshin and A. Tanguy, Boson peak and Ioffe-Regel criterion in amorphous silicon-like materials: effect of bond directionality, Phys. Rev. E, 2016, 93, 023006.

24 C. Weigel, M. Foret, B. Hehlen, M. Kint, S. Clément, A. Polian, R. Vacher and B. Rufflé, Polarized raman spectroscopy of $v-\mathrm{SiO}_{2}$ under rare-gas compression. Phys. Rev. B, 2016, 93, 224303.

25 S. Sen, H. Maekawa and G.N. Papatheodorou, Short-range structure of invert glasses along the pseudo-binary join $\mathrm{MgSiO}_{3}-\mathrm{Mg}_{2} \mathrm{SiO}_{4}$ : results from ${ }^{29} \mathrm{Si}$ and ${ }^{25} \mathrm{Mg}$ MAS NMR spectroscopy. J. Phys. Chem. B, 2009, 113, 15243-15248.

$26 \mathrm{~L}$. Cormier and G.J. Cuello, $\mathrm{Mg}$ coordination in a $\mathrm{MgSiO}_{3}$ glass using neutron diffraction coupled with isotopic substitution. Phys. Rev. B, 2011, 83, 224204.

27 C.T. Kirk, Quantitative analysis of the effect of disorderinduced mode coupling on infrared absorption in silica. Phys. Rev. B, 1988, 38, 1255.

28 A.H. Dietzel, On the so-called mixed alkali effect. Phys. chem. Glas. Phys. Chem. Glas., 1983, 23, 172-180.

29 V. Magnien, D.R. Neuville, L. Cormier, J. Roux, J.-L. Hazemann, D. de Ligny, S. Pascarelli, I. Vickridge, O. Pinet P. and Richet, Kinetics and mechanisms of iron redox reactions in silicate melts: The eects of temperature and alkali cations. Geochim. Cosmochim. Acta, 2008, 72, 2157-2168.

30 C. Le Losq, D.R. Neuville, P. Florian, G.S. Henderson and D. Massiot, The role of $\mathrm{Al}^{3+}$ on rheology and structural changes in sodium silicate and aluminosilicate glasses and melts. Geochimica et Cosmochimica Acta, 2014, 126, 495-517.

31 M.R. Cicconi, D. de Ligny, T.M. Gallo and D.R. Neuville, Ca Neighbors from XANES spectroscopy: a tool to investigate structure, redox and nucleation processes in silicate glasses, melts and crystals. American Mineralogist, 2016, 101, 12321236.

32 Y.D. Yiannopoulos, G.D. Chryssikos and E.I. Kamitsos, Structure and properties of alkaline earth borate glasses. Phys. Chem. Glasses, 2001, 42, 164-72.

33 W.J. Dell, P.J. Bray and S.Z. Xiao, ${ }^{11} \mathrm{~B}$ NMR studies and structural modeling of $\mathrm{Na}_{2} \mathrm{O}-\mathrm{B}_{2} \mathrm{O}_{3}-\mathrm{SiO}_{2}$ glasses of high soda content. J. non-Cryst. Solids 1983, 58, 1-16. 